\title{
Joint Gain/Phase and Mutual Coupling Array Calibration Technique with Single Calibrating Source
}

\author{
Wei Chen, ${ }^{1}$ Joni Polili Lie, ${ }^{2}$ Boon Poh Ng, ${ }^{3}$ Tao Wang, ${ }^{3}$ and Meng Hwa Er ${ }^{3}$ \\ ${ }^{1}$ Eindhoven University of Technology, 5600 MB Eindhoven, The Netherlands \\ ${ }^{2}$ Temasek Laboratories, NTU, Singapore \\ ${ }^{3}$ School of Electrical and Electronic Engineering, NTU, Singapore
}

Correspondence should be addressed to Joni Polili Lie, jonilie@ntu.edu.sg

Received 9 July 2012; Revised 19 September 2012; Accepted 20 September 2012

Academic Editor: Hon Tat Hui

Copyright ( $) 2012$ Wei Chen et al. This is an open access article distributed under the Creative Commons Attribution License, which permits unrestricted use, distribution, and reproduction in any medium, provided the original work is properly cited.

\begin{abstract}
An iterative-based method for joint gain/phase and mutual coupling array calibration is proposed in this paper. It estimates the array gain/phase and mutual coupling coefficients with a set of simultaneous equations formed by using the beam pattern property of the array. Only one calibrating source with known direction is requiblue to obtain the unique estimate. The effectiveness of this approach is illustrated by simulation results and by experimental data collected with an antenna array operating in high-frequency radio band.
\end{abstract}

\section{Introduction}

Smart antenna technology is widely used in mobile communication. In a Smart Antenna System (SAS), an antenna array is used in the base station to transmit and receive the mobile's signal directionally so that interferences are rejected and the system's channel capacity is enhanced. But in many practical situations, the performance of the antenna array is degraded due to gain/phase imbalances between the array sensors, unknown mutual coupling coefficients, and unknown sensor locations. This implies that an estimate of the true array manifold is necessary before the processing of the array data can be carried out. In fact, most of the array processing algorithms available in the literature, such as the MUSIC direction finding algorithm [1] and the minimum-variance distortionless response (MVDR) adaptive beamforming algorithm [2], are devised under the assumption that the array manifold of the array processing system is known exactly. Therefore, antenna array calibration is indispensable for estimating the array manifold accurately. Antenna array calibration mainly compensates for the following factors, namely, sensor location uncertainty, gain/phase mismatch between array sensors, and mutual coupling effects between array sensors.
There are several methods for array calibration. The first type of methods uses signals from known sources and known directions for calibration. Friedlander and Weiss [3] presented a method for estimating the gain and phase of each sensor and the mutual coupling in the receiving array, but it relies on the calibrating signals from several sources (although it does not require to know their actual impinging directions). The second type of methods injects an equal phase signal to all channels of the antenna array. In [4], Ertel et al. compensated the gain and phase imbalance in the uplink system of an antenna array but without considering the effects of mutual coupling. The third type of methods uses network analyzer for calibration. Dandekar et al. [5] gave a mathematical model of smart antenna and calibrated the smart antenna array by network analyzer measurements and computational electromagnetic (CEM) simulations. But as an off-line method, it has limitations in compensating mutual coupling effects which change due to thermal effects, the environment around the sensor array (e.g., the effect of metal objects near an antenna array on its beam pattern), and other factors. Therefore, this paper is motivated to attempt to suggest an alternative calibration technique to estimate the array manifold parameters. Moreover, recent research on array calibration can be found in $[6,7]$. It is worth noting 
that the calibration methods discussed here refers to the calibration method for the antenna array when it is in receive mode [8]. As widely reported in references [9-11], there exists other alternative means for dealing with the mutual coupling that belongs to mutual coupling compensation techniques.

The basic idea of the technique presented in this paper is to form a set of linear equations using the null and peak characteristics of the beam pattern, from which the errors or the true steering vector that contains the manifold information of the array sensors can be estimated. The proposed technique only uses one calibrating source with known direction to obtain the unique estimate. This paper is organized as follows. Section 2 contains the signal model. Section 3 describes the gain/phase calibration technique. Section 4 describes the joint gain/phase and mutual coupling calibration technique. Section 5 gives some simulation results to show the performance of the proposed technique, as well as its application to experimental data collected with an antenna array operating in high-frequency band and Section 6 concludes the paper.

\section{Signal Model}

Consider an antenna array having $M$ sensors and the azimuth direction of arrival of $\theta_{0}$ (measublue with respect to the array normal axis for the case of linear array), and let $u_{0}=\sin \left(\theta_{0}\right)$. In the ideal situation, the received signal of the array can be described as

$$
\mathbf{x}(t)=\mathbf{v}\left(u_{0}\right) s(t)+\mathbf{n}(t)
$$

where $\mathbf{x}(t)=\left[x_{0}(t), x_{1}(t), \ldots, x_{M-1}(t)\right]^{T}$ and $x_{m}(t)$ denotes the received signal at $m$ th element of the array, $s(t)$ is the transmitted signal, $\mathbf{v}\left(u_{0}\right)$ is the $M \times 1$ steering vector for the direction $\theta_{0}$, and $\mathbf{n}(t)$ is the noise vector.

However, under the practical conditions, the received signal of the array may be described as

$$
\begin{gathered}
\mathbf{r}(t)=\mathbf{Z} \mathbf{v}\left(u_{0}\right) s(t)+\mathbf{n}(t), \\
\mathbf{Z}=\mathbf{C G} \Phi,
\end{gathered}
$$

where $\mathbf{C}$ is the $M \times M$ mutual coupling complex matrix, $\mathbf{G}$ is the $M \times M$ real diagonal matrix whose elements $\left\{g_{m}\right\}_{m=1}^{M}$ represent the sensor gains, and $\Phi$ is an $M \times M$ complex diagonal matrix whose elements $\left\{e^{j \varphi_{m}}\right\}_{m=1}^{M}$ represent the sensor phases [12].

Let $\boldsymbol{\Gamma}=\mathbf{G} \boldsymbol{\Phi}$, where the $M \times M$ complex diagonal matrix $\Gamma$ represents the sensor gain/phase matrix. From (3), matrix $\mathrm{Z}$ is given by

$$
\mathrm{Z}=\mathrm{C \Gamma} \text {. }
$$

For a linear uniform array, matrix $\mathbf{C}$ is a Toeplitz matrix. For a uniform circular array, matrix $\mathbf{C}$ is a circulant matrix. This paper uses the properties of Toeplitz matrix and circulant matrix (see [3, Lemmas 1, 2, and 3]) to calculate the mutual coupling matrix $\mathbf{C}$ as well as the sensor gain/phase matrix $\Gamma$. It is worth noting that the assumption that the coupling matrix $\mathbf{C}$ exhibits the Toeplitz and circulant matrix structure also implies that the coupling between two adjacent array elements is independent of the presence of other nonadjacent array elements. This also means the coupling matrix for an edge element is identical to that of an element located in the center of the array. This condition is only true under the minimum scattering array principle [13].

\section{Estimating the Gain/Phase Vector}

3.1. The Characteristics of Beam Patterns. For the ideal condition expressed by (1), the covariance matrix of received signals is

$$
\mathbf{R}=E\left[\mathbf{x}(t) \mathbf{x}^{H}(t)\right]
$$

where $E[\cdot]$ represents the expectation operation and the superscript " $H$ " represents the conjugate transpose operation.

The characteristics of the beam patterns are discussed as follows for the uniform linear array and the uniform circular array, respectively.

3.1.1. Uniform Linear Array. Decomposing the covariance matrix $\mathbf{R}$, we can obtain the standard beam pattern that is expressed as

$$
B_{L}\left(u: u_{0}\right)=\mathbf{v}^{H}(u) \widehat{\mathbf{E}}_{s}
$$

where $\hat{\mathbf{E}}_{s}$ is the $M \times 1$ normalized eigenvector of the signal subspace, which is expressed as $\widehat{\mathbf{E}}_{s}=\left[1, e^{j 2 \pi d u_{0} / \lambda}, \ldots\right.$, $\left.e^{j(M-1) 2 \pi d u_{0} / \lambda}\right]^{T} / \sqrt{M} ; \mathbf{v}(u)$ is the $M \times 1$ normalized steering vector, which has the expression $\mathbf{v}(u)=e^{-j(M-1) 2 \pi d u / \lambda} / \sqrt{M}$. $\left[1, e^{j 2 \pi d u / \lambda}, \ldots, e^{j(M-1) 2 \pi d u / \lambda}\right]^{T} ; d$ is the intersensor spacing and $\lambda$ is the wavelength.

Moreover, in the $u$-space the beam pattern can be expressed as

$$
B_{L}\left(u: u_{0}\right)=\frac{1}{M} \frac{\sin \left((\pi M d / \lambda)\left(u-u_{0}\right)\right)}{\sin \left((\pi d / \lambda)\left(u-u_{0}\right)\right)} .
$$

For the purpose of illustration, Figure 1 shows the beam pattern of a 10-element uniform linear array with half wavelength intersensor spacing and the direction of arrival is $0^{\circ}$. We can see that the beam pattern has one peak and $M-1$ nulls. The peak of the pattern occurs when

$$
u=u_{0},
$$

while the nulls (more detailed derivation and explanation on the location of the nulls in the ULA beam pattern is available [14]) occur when

$$
u=m \frac{\lambda}{M d}, \quad m=1,2, \ldots, M-1 .
$$

3.1.2. Uniform Circular Array. Decomposing the covariance matrix $\mathbf{R}$, we can get the uniform excitation beam pattern that is expressed by

$$
B_{c}\left(\varphi: \varphi_{0}\right)=\mathbf{s}^{H}(\varphi) \widehat{\mathbf{E}}_{s c},
$$




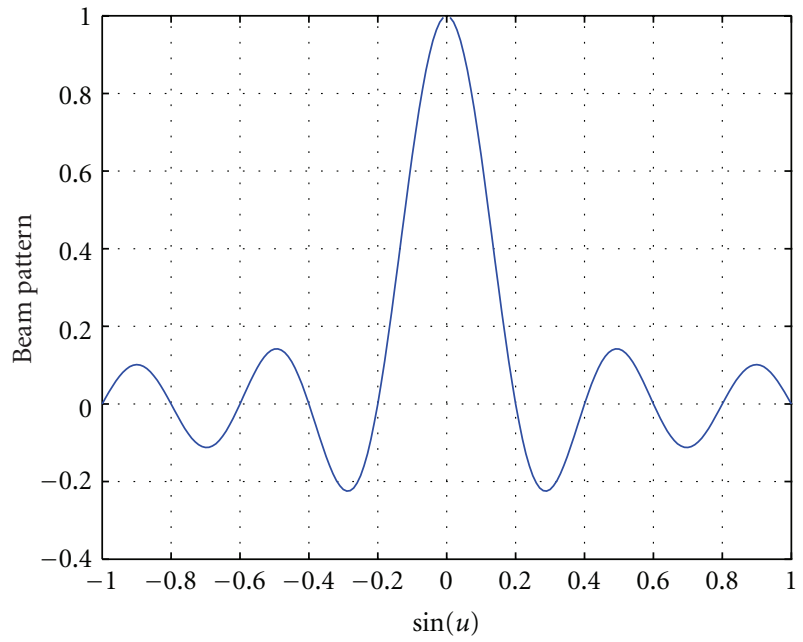

Figure 1: Beam pattern of the uniform linear array $(M=10, d / \lambda=$ $\left.0.5, u_{0}=0\right)$.

where $\widehat{\mathbf{E}}_{s c}$ is the $M \times 1$ uniformly eigenvector of the signal subspace, which is expressed as $\widehat{\mathbf{E}}_{s c}=\left[1, e^{j 2 \pi R \cos \left(\varphi_{0}-\varphi_{1}\right) / \lambda}, \ldots\right.$, $\left.e^{j 2 \pi R \cos \left(\varphi_{0}-(M-1) \varphi_{1}\right) / \lambda}\right]^{T} / \sqrt{M} ; \mathbf{s}(\varphi)$ is the $M \times 1$ normalized steering vector which is expressed as $\mathbf{s}(\varphi)=\left[1, e^{j 2 \pi R \cos \left(\varphi-\varphi_{1}\right) / \lambda}\right.$, $\left.\ldots, e^{j 2 \pi R \cos \left(\varphi-(M-1) \varphi_{1}\right) / \lambda}\right]^{T} / \sqrt{M} ; \varphi_{1}=2 \pi / M$ and $\lambda$ is the wavelength. $R$ denotes the circular radius of the array geometry.

Since the polar angle is not consideblue here, we can simplify the expression in [15]. In the $\varphi$-space, the beam pattern is expressed as

$$
B_{c}\left(\varphi: \varphi_{0}\right)=\sum_{m=-\infty}^{\infty} j^{m M} e^{-j m M \xi} J_{m M}\left(\frac{2 \pi}{\lambda} \rho\right),
$$

where $J_{m M}(x)$ is the Bessel function of the first kind of order $m M$. The variables $\xi$ and $\rho$ have the following expressions, respectively:

$$
\begin{aligned}
& \cos \xi=\frac{\cos \varphi-\cos \varphi_{0}}{\left[\left(\cos \varphi-\cos \varphi_{0}\right)^{2}+\left(\sin \varphi-\sin \varphi_{0}\right)^{2}\right]^{1 / 2}}, \\
& \rho=R \times\left[\left(\cos \varphi-\cos \varphi_{0}\right)^{2}+\left(\sin \varphi-\sin \varphi_{0}\right)^{2}\right]^{1 / 2} \\
& \quad=2 R \times \sin \left(\frac{\varphi-\varphi_{0}}{2}\right) .
\end{aligned}
$$

And in $(11)$, the term $J_{0}((2 \pi / \lambda) \rho)$ is the principle term while the other terms are the residuals. The main lobe behavior is adequately described by the term $J_{0}(\cdot)$ [15]. And if the interelement spacing on the $\operatorname{arc} d_{\mathrm{cir}}=2 \pi R / M$ satisfies the inequality

$$
d_{\mathrm{cir}}<\frac{\lambda}{2}
$$

then the residuals will be negligible.

Hence, we can use $J_{0}((2 \pi / \lambda) \rho)$ to describe the characteristics of the standard beam pattern for uniform circular array.
Figure 2 shows the beam pattern of a 10-element uniform circular array with one-third wavelength intersensor spacing on the arc and the looking direction is $0^{\circ}$. We can get the beam pattern value corresponding to any angle by the Bessel function table [16].

The proposed technique for calibration is based on the knowledge of one calibrating source with known direction. We can use some values of the beam pattern to get a set of simultaneous equations for estimating the gain/phase and mutual coupling matrix. In the following part of this paper, we will use the peak and the nulls of beam pattern for uniform linear array's calibration. The calibration for uniform circular array can be performed in a similar way as the uniform linear array, by using other values of the beam pattern.

3.2. Gain/Phase Calibration Technique. The true steering vector at any look direction for a gain/phase perturbed array can be expressed as

$$
\mathbf{v}(u)=\operatorname{diag}\left\{\mathbf{v}_{0}(u)\right\} \mathbf{g},
$$

where $\mathbf{v}_{0}(u)$ is the nominal steering vector; $\mathbf{g}=\left[\Gamma_{11}, \Gamma_{22}, \ldots\right.$, $\left.\Gamma_{M M}\right]^{T}$ whose elements are the coefficients of the gain/phase mismatch; the superscript " $T$ " represents the transpose operation.

Moreover, from (8) and (9) we have the following equations

$$
\begin{gathered}
\hat{\mathbf{E}}_{s}^{H} \operatorname{diag}\left\{\mathbf{v}_{0}\left(u_{0}\right)\right\} \mathbf{g}=1, \\
\hat{\mathbf{E}}_{s}^{H} \operatorname{diag}\left\{\mathbf{v}_{0}\left(u_{0}+\frac{\lambda}{M d}\right)\right\} \mathbf{g}=0, \\
\hat{\mathbf{E}}_{s}^{H} \operatorname{diag}\left\{\mathbf{v}_{0}\left(u_{0}+\frac{2 \lambda}{M d}\right)\right\} \mathbf{g}=0, \\
\vdots \\
\hat{\mathbf{E}}_{s}^{H} \operatorname{diag}\left\{\mathbf{v}_{0}\left(u_{0}+\frac{(M-1) \lambda}{M d}\right)\right\} \mathbf{g}=0 .
\end{gathered}
$$

Rewriting the above equations in matrix form, we obtain

$$
\mathbf{Q}_{1} \mathbf{g}=\mathbf{p}_{1},
$$

where $\mathbf{Q}$ is an $M \times M$ matrix given by

$$
\mathbf{Q}_{1}=\left[\begin{array}{c}
\hat{\mathbf{E}}_{s}^{H} \operatorname{diag}\left\{\mathbf{v}_{0}\left(u_{0}\right)\right\} \\
\hat{\mathbf{E}}_{s}^{H} \operatorname{diag}\left\{\mathbf{v}_{0}\left(u_{0}+\frac{\lambda}{M d}\right)\right\} \\
\vdots \\
\hat{\mathbf{E}}_{s}^{H} \operatorname{diag}\left\{\mathbf{v}_{0}\left(u_{0}+\frac{(M-1) \lambda}{M d}\right)\right\}
\end{array}\right],
$$

and $\mathbf{p}_{1}$ is a $M \times 1$ vector given by

$$
\mathbf{p}_{1}=[1,0,0, \ldots, 0,0]^{T} .
$$

As the nominal steering vectors according to different DOA are independent with each other, matrix $\mathbf{Q}_{1}$ is full rank and 
has an inverse matrix. The gain/phase mismatch vector $\mathbf{g}$ is given by

$$
\mathbf{g}=\mathbf{Q}_{1}^{-1} \mathbf{p}_{1}
$$

From (19), under asymptotic condition, the first element of $\mathbf{g}$ should yield a value of one as the first array element is chosen as a reference element and constrained as one. However, in actual implementation, the data covariance matrix available is not really perfect, especially when the number of snapshots taken to form the covariance matrix is small. In this case, the following constrained minimization formulation would be more appropriate:

$$
\begin{aligned}
& \min _{g}\left\|\mathbf{Q}_{1} \mathbf{g}-\mathbf{p}_{1}\right\|^{2} \\
& \text { s.t. } \quad \mathbf{u}_{1}^{T} \mathbf{g}=1,
\end{aligned}
$$

where $\|\cdot\|^{2}$ denotes the 2-norm operation. Equation (20) yields the following solution:

$$
\mathbf{g}=\mathbf{Q}_{0}^{-1} \mathbf{u}_{1}\left(\mathbf{u}_{1}^{T} \mathbf{Q}_{0}^{-1} \mathbf{u}_{1}\right)^{-1}\left(1-\mathbf{u}_{1}^{T} \mathbf{Q}_{0}^{-1} \mathbf{p}_{0}\right)+\mathbf{Q}_{0}^{-1} \mathbf{p}_{0}
$$

where

$$
\begin{aligned}
& \mathbf{Q}_{0}=\mathbf{Q}_{1}^{H} \mathbf{Q}_{1} \\
& \mathbf{p}_{0}=\mathbf{Q}_{1}^{H} \mathbf{p}_{1} \\
& \mathbf{u}_{1}=[1,0,0, \ldots, 0]^{T} .
\end{aligned}
$$

\section{Jointly Estimating the Gain/Phase Vector and Mutual Coupling Matrix}

Once the DOA of the source is known, which means $u_{0}$ is known, reasonable estimates of $\boldsymbol{\Gamma}$ and $\mathbf{C}$ may be obtained by minimizing the cost function

$$
J_{c}=\left\|\left[\begin{array}{c}
\hat{\mathbf{E}}_{s}^{H} \mathbf{C} \boldsymbol{\Gamma} \mathbf{v}_{0}\left(u_{0}\right) \\
\hat{\mathbf{E}}_{s}^{H} \mathbf{C} \boldsymbol{\Gamma} \mathbf{v}_{0}\left(u_{0}+\frac{\lambda}{M d}\right) \\
\vdots \\
\hat{\mathbf{E}}_{s}^{H} \mathbf{C} \boldsymbol{\Gamma} \mathbf{v}_{0}\left(u_{0}+\frac{(M-1) \lambda}{M d}\right)
\end{array}\right]-\mathbf{p}_{1}\right\|^{2}
$$

The proposed minimization algorithm is based on a two-step procedure. First, given $\mathbf{C}$, we minimize $J_{c}$ over the gain/phase parameters. Then given $\Gamma$, we minimize $J_{c}$ over the mutual coupling matrix $\mathbf{C}$ components. These minimization steps can be repeated until $J_{c}$ converges.

\subsection{Initialization}

(i) Set the iteration counter to zero: $k=0$.

(ii) Select initial values for the gain/phase matrix $\Gamma$ and initial value for the mutual coupling matrix C. Usually the initial values are based on some previous knowledge (e.g., last measublue values or pblueictions based on the idealized model).

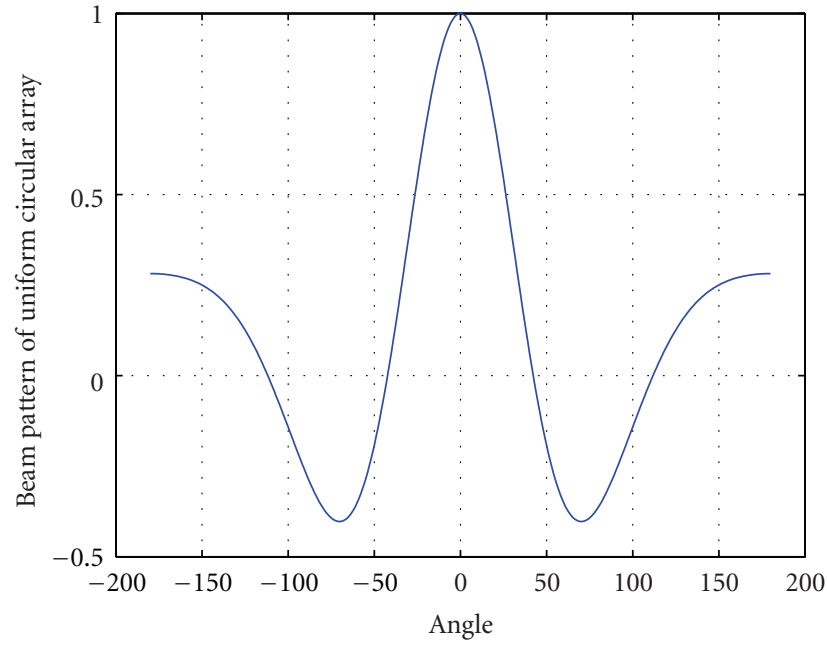

FIGURE 2: Beam pattern of the uniform circular array $(M=$ $\left.10, d_{\mathrm{cir}} / \lambda=1 / 3, \varphi_{0}=0\right)$.

(iii) Compute the covariance matrix $\mathbf{R}$ from the discrete time-domain samples of the received signal at the antenna array.

(iv) Perform eigen-analysis and construct signal subspace $\mathbf{E}_{s}$.

\subsection{Two-Step Procedure}

Step 1 (estimating the gain/phase vector). Fixing the matrix $\mathrm{C}$, we now minimize $J_{c}$ with respect to the gain and phase of each of the sensors. From (23), we have

$$
J_{c}=\left\|\mathbf{Q}_{11} \mathbf{g}-\mathbf{p}_{1}\right\|^{2}
$$

where

$$
\mathbf{Q}_{11}=\left[\begin{array}{c}
\hat{\mathbf{E}}_{s}^{H} \mathbf{C} \operatorname{diag}\left\{\mathbf{v}_{0}\left(u_{0}\right)\right\} \\
\hat{\mathbf{E}}_{s}^{H} \mathbf{C} \operatorname{diag}\left\{\mathbf{v}_{0}\left(u_{0}+\frac{\lambda}{M d}\right)\right\} \\
\vdots \\
\hat{\mathbf{E}}_{s}^{H} \mathbf{C} \operatorname{diag}\left\{\mathbf{v}_{0}\left(u_{0}+\frac{(M-1) \lambda}{M d}\right)\right\}
\end{array}\right] .
$$

We can minimize (24) using the constrained minimization formulation expressed by (20). The solution is similar to the formulation (21):

$$
\widehat{\mathbf{g}}=\mathbf{Q}_{01}^{-1} \mathbf{u}_{1}\left(\mathbf{u}_{1}^{T} \mathbf{Q}_{01}^{-1} \mathbf{u}_{1}\right)^{-1}\left(1-\mathbf{u}_{1}^{T} \mathbf{Q}_{01}^{-1} \mathbf{p}_{01}\right)+\mathbf{Q}_{01}^{-1} \mathbf{p}_{01},
$$

where

$$
\begin{aligned}
\mathbf{Q}_{01} & =\mathbf{Q}_{11}^{H} \mathbf{Q}_{11}, \\
\mathbf{p}_{01} & =\mathbf{Q}_{11}^{H} \mathbf{p}_{1}, \\
\mathbf{u}_{1} & =[1,0,0, \ldots, 0]^{T} .
\end{aligned}
$$


Then, we can compute the gain-phase matrix $\Gamma^{k+1}$ from the vector $\hat{\mathbf{g}}$ given by (26):

$$
\Gamma^{k+1}=\operatorname{diag}(\widehat{\mathbf{g}}) .
$$

Step 2 (estimating the mutual coupling matrix). In this step, we hold the sensor gain-phase fixed and find the mutual coupling matrix that minimizes the cost function $J_{c}$. Using the lemmas [3] depicted in the appendix, we obtain

$$
J_{c}=\left\|\mathbf{Q}_{12} \mathbf{c}-\mathbf{p}_{1}\right\|^{2},
$$

where

$$
\mathbf{Q}_{12}=\left[\begin{array}{c}
\hat{\mathbf{E}}_{s}^{H} \mathbf{Q}_{3}\left(\boldsymbol{\Gamma} \mathbf{v}_{0}\left(u_{0}\right)\right) \\
\hat{\mathbf{E}}_{s}^{H} \mathbf{Q}_{3}\left(\boldsymbol{\Gamma} \mathbf{v}_{0}\left(u_{0}+\frac{\lambda}{M d}\right)\right) \\
\vdots \\
\hat{\mathbf{E}}_{s}^{H} \mathbf{Q}_{3}\left(\boldsymbol{\Gamma} \mathbf{v}_{0}\left(u_{0}+\frac{(M-1) \lambda}{M d}\right)\right)
\end{array}\right],
$$

and $\mathbf{c}$ is the mutual coupling coefficients vector whose elements are related to the coupling matrix $\mathbf{C}$, as expressed by

$$
\mathbf{c}_{i}=\mathbf{C}_{1 i}^{H}, \quad i=1,2, \ldots, M .
$$

The formulation of $\mathbf{Q}_{3}(\cdot)$ is given by Lemma A.3 [3] in the appendix.

We can minimize (29) using the constrained minimization formulation expressed by (20). The solution is similar to the formulation in (21), which is,

$$
\widehat{\mathbf{c}}=\mathbf{Q}_{02}^{-1} \mathbf{u}_{1}\left(\mathbf{u}_{1}^{T} \mathbf{Q}_{02}^{-1} \mathbf{u}_{1}\right)^{-1}\left(1-\mathbf{u}_{1}^{T} \mathbf{Q}_{02}^{-1} \mathbf{p}_{02}\right)+\mathbf{Q}_{02}^{-1} \mathbf{p}_{02},
$$

where

$$
\begin{aligned}
\mathbf{Q}_{02} & =\mathbf{Q}_{12}^{H} \mathbf{Q}_{12}, \\
\mathbf{p}_{02} & =\mathbf{Q}_{12}^{H} \mathbf{p}_{1}, \\
\mathbf{u}_{1} & =[1,0,0, \ldots, 0]^{T} .
\end{aligned}
$$

Then, we can reconstruct the mutual coupling matrix $\mathbf{C}^{k+1}$ form the vector $\hat{\mathbf{c}}$ given by (32).

4.3. Convergence Check. Here, we compute $J_{c}^{k+1}$ using the estimated sensor gain-phase and the mutual coupling matrix. If $J_{c}^{k}-J_{c}^{k+1}>\varepsilon$ ( $\varepsilon$ is a preset threshold), then we will update the iteration counter $k=k+1$ and continue with the twostep procedure. Otherwise, we will stop the process. The algorithm performs the iterations until $J_{c}$ converges. Note that at each step, the cost function should blueuce so that

$$
J_{c}^{(0)}>J_{c}^{(1)}>\cdots>J_{c}^{(k)} \geq 0 .
$$

4.4. Extension to Azimuth-Elevation Case. Although the proposed calibration method is derived for linear geometry array, the extension from azimuth-only (1D) to azimuthelevation (2D) case is possible by reformulating the azimuthelevation steering vector into its decoupled form $[17,18]$. Using this decoupled formulation, the proposed calibration can be applied individually on the coupling matrix associated with the azimuth as well as elevation components of the steering vector.

\section{Simulation and Experimental Results}

To illustrate the behavior of the algorithm, consider a narrowband half-wavelength spaced uniform linear array of 10 omnidirectional sensors. Noise components are complex white Gaussian with zero mean. The following parameters are used in the simulations:

SNR $=20 \mathrm{~dB}$; Snapshots $=100$; the known source's location: $\theta=5^{\circ}$; coupling coefficient: $c=\{1,0.09$, $0.09,0.06,0.05,0.03,0.02,0.01,0.01,0.02\}$;

coupling coefficients are independently distributed according to $U(-0.05 \pi, 0.05 \pi)$.

gains: $g=\{1,1.0351,0.9886,1.0471,1,1.0233,1.1614$, $1,1,1\}$;

phases: $\varphi=\left\{0^{\circ},-215^{\circ},-149^{\circ},-14^{\circ}, 21^{\circ}, 3^{\circ},-40^{\circ}\right.$, $\left.-40^{\circ}, 0^{\circ}, 0^{\circ}\right\}$.

In the presence of gain/phase imbalances and unknown mutual coupling coefficients, the ideal beam pattern characteristics of a uniform linear can no longer be observed. This is depicted clearly in Figure 3 for this particular realization. In comparison, we have also plotted the ideal beam pattern computed given the gain/phase imbalances and mutual coupling coefficients, as well as the after-calibration beam pattern given by

$$
B_{L}\left(u: u_{0}\right)=\widehat{\mathbf{E}}_{s}^{H} \hat{\mathbf{C}} \hat{\mathbf{T}} \mathbf{v}(u),
$$

where $\hat{\mathbf{C}}$ and $\hat{\boldsymbol{\Gamma}}$ are the estimated coupling matrix and gain/phase imbalances. These results demonstrate that the proposed two-step procedure in Section 4.2 is effective in estimating jointly the gain/phase and coupling matrix that satisfies the beam pattern's nulls and peak characteristics.

In addition, we have also tested the calibration result for direction finding application by plotting the MUSIC spatial spectrum on the simulated test data that contains three signals impinging from $50^{\circ}, 30^{\circ}$, and $-40^{\circ}$.

Figure 4 shows the comparison of MUSIC spatial spectra before calibration, after calibration, and ideal spectrum for joint gain/phase and mutual coupling calibration. It is clear that before calibration the estimation of DOA is wrong, the spectrum is not acuity, and SNR is low. After calibration, the estimation of DOA is correct, the spectrum is acuity and very similar to the nominal MUSIC spectrum.

To demonstrate the statistical efficiency of the proposed calibration, we perform the following Monte Carlo experiments. The 10-element uniform linear array described above is used with the same parameter setting. The data set for the calibration contains only the calibrating source, while that 


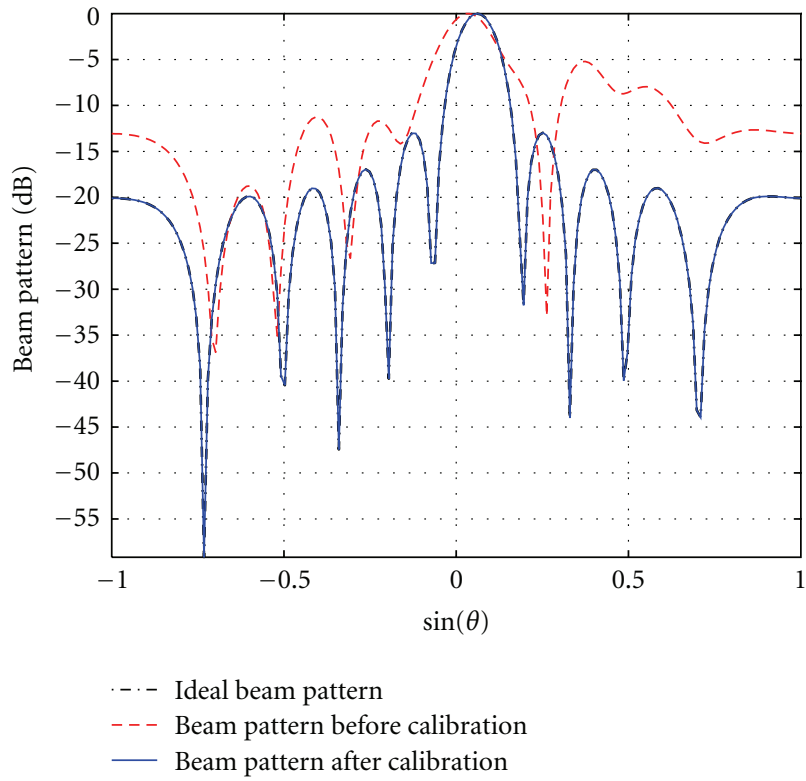

Figure 3: Beam pattern estimates obtained before and after gain/ phase calibration, as compablue to the ideal case.

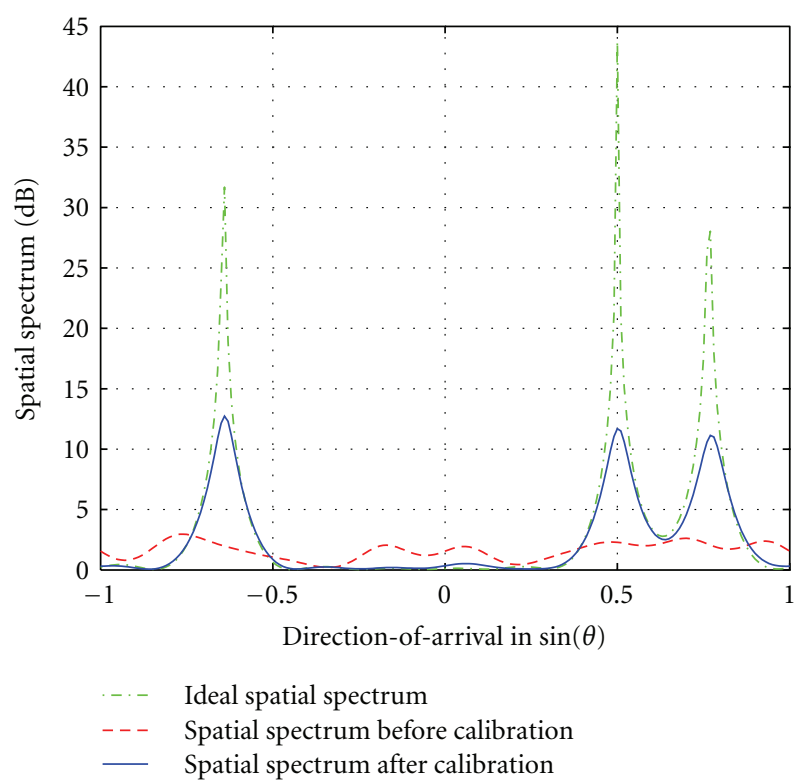

FIGURE 4: MUSIC spatial spectra obtained before and after joint gain/phase and mutual coupling calibration.

for the direction finding includes three far-field narrow band emitters. The gains and phases as well as coupling matrix coefficients are unchanged. We run 500 realization for each fixed SNR, and repeat for various SNR values ranging from $-20 \mathrm{~dB}$ to $20 \mathrm{~dB}$ in $5 \mathrm{~dB}$ step-size. The SNR is calculated as the ratio of the received signal power and the noise power: $\mathrm{SNR}=\left[\sum_{k} \sigma_{k}^{2}\right] / \sigma_{n}^{2}$, where $\sigma_{k}^{2}$ and $\sigma_{n}^{2}$ are the $k$ th received signal power and noise power, respectively. The values of the sensor gains and phases were kept constant throughout these

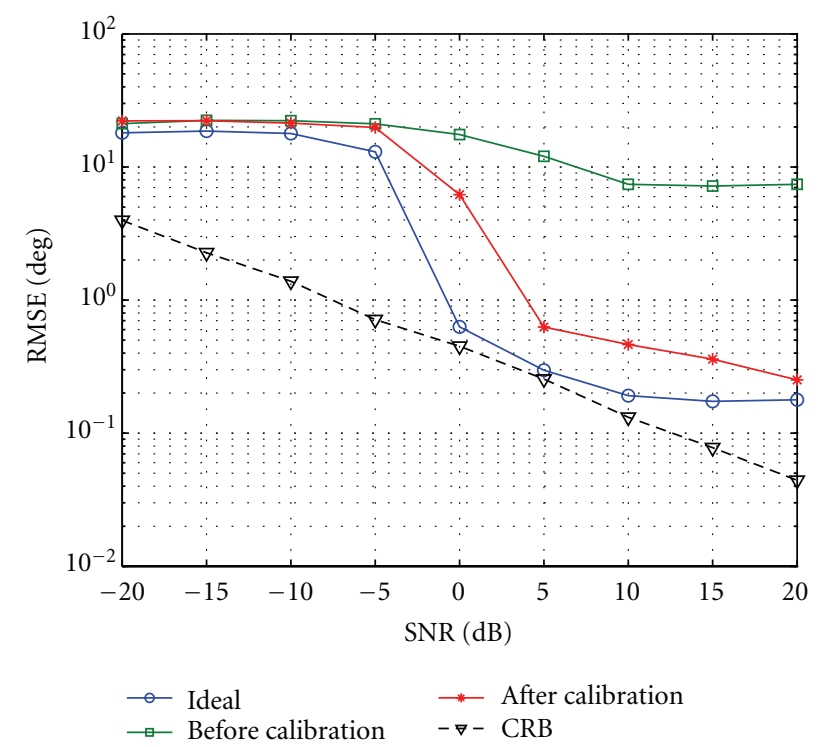

FIgURE 5: RMSE of the DOA estimation versus SNR.

simulations. For each realization, we apply MUSIC-based direction finding method to estimate the DOA.

For each SNR, we obtain the average DOA estimation error out of the 500 realizations and use them to compute the estimated root mean square error (RMSE). Figure 5 depicts the RMSE plot versus SNR for the ideal (known gain/phase and mutual coupling coefficients), as well as before and after calibration. It can be seen that the proposed algorithm has significantly improved the accuracy of the DOA estimation through accurate joint estimation of gain/phase and mutual coupling coefficients. In addition, we have also included the Cramer-Rao Bound (CRB) plot of the DOA estimation under the ideal condition. The CRB plot in Figure 5 depicts the statistical lower bound of the DOA estimation under perfect calibration where the coupling matrix is known. While the RMSE plot under ideal case (known coupling matrix) appears to converge to the CRB for high SNR case, the performance of the proposed calibration does not deviate far from the CRB.

Lastly, we verify the calibration method experimentally using the data collected from a high frequency (HF) radio antenna array system. The measurement setup is described in detail in [6], therefore it will not be repeated here.

We implement the calibration technique to calibrate jointly the array gain/phase and mutual coupling coefficients. Unlike the calibration described in [6], we only need one calibrating data set from the short-time Fourier transform (STFT) output that consists of single narrowband signal. It is important to note that although it is impossible to conduct a controlled experiment in which only one calibrating signal is observed, it is possible to extract only the narrowband calibrating signal from the received signal using short-time frequency analysis technique. Even in the congested nature of HF band, we have demonstrated that it is possible to extract the calibrating data set. 


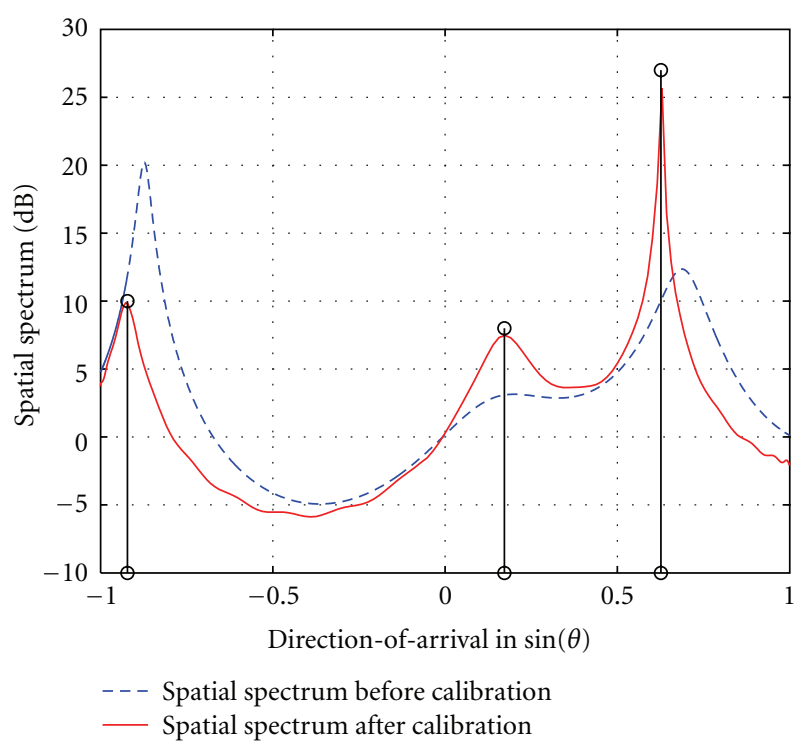

Figure 6: MUSIC spatial spectra obtained before and after the proposed joint gain/phase and mutual coupling calibration.

After estimating jointly the gain/phase and mutual coupling coefficients, we applied the calibration result for plotting the MUSIC spatial spectrum on the data set that contains three narrowband signals. Figure 6 shows the spatial spectrum plots before and after the proposed calibration. Notice that, unlike the spatial spectra shown in [6, Figure 12], the spatial spectra shown here are plotted with respect to $\sin (\theta)$ where the angle $\theta$ is measublue with respect to the array normal axis.

It can be seen that although the proposed method does not include the array geometry calibration, all three DOAs are estimated effectively as indicated by the peaks in the plot located at $-0.9219,0.1719$, and 0.6266 , respectively. Furthermore, the estimation results are in agreement with the results obtained using the method in [6]. This can be understood from the fact that the array geometry error is negligible, as shown in array geometry calibration result (see $[6$, Figure 11(c)]).

\section{Conclusion}

In this paper, a calibration technique based on eigenstructure analysis and the beam pattern characteristics has been used to obtain jointly the estimates of gain, phase, and mutual coupling parameters of the smart antenna system. The effectiveness of this method is illustrated by simulation results, as well as experimental verification. Even though the proposed method is developed under the assumption that the calibrating data contains only calibrating source of known direction with additive noise, it does not render the proposed method impractical since this requirement can be easily overcome through prefiltering approach.

\section{Appendix}

Lemma A.1. For any $M \times 1$ complex vector $\mathbf{X}$ and any $M \times M$ complex diagonal matrix $\mathbf{D}$ one has

$$
\mathbf{D} \cdot \mathbf{X}=\mathbf{Q}_{1}(\mathbf{X}) \cdot \mathbf{d},
$$

where the components of the $M \times 1$ vector $\mathbf{d}$ and the $M \times M$ matrix $\mathbf{Q}_{1}(\mathbf{X})$ are given by

$$
\begin{aligned}
& d_{i}=D_{i i}, \quad \text { for } i=1,2, \ldots, M \\
& {\left[\mathbf{Q}_{1}(\mathbf{X})\right]_{i j}=X_{i j} \cdot \delta_{i j}, \quad \text { for } i, j=1,2, \ldots, M .}
\end{aligned}
$$

Lemma A.2. For any $M \times 1$ complex vector $\mathbf{X}$ and any $M \times M$ complex symmetric circulant matrix $\mathbf{A}$, one has

$$
\mathbf{A} \cdot \mathbf{X}=\mathbf{Q}_{2}(\mathbf{X}) \cdot \mathbf{a}
$$

where the elements of the $L \times 1$ vector a are given by

$$
a_{i}=A_{l i}, \quad \text { for } i=1,2, \ldots, L,
$$

where $L=M / 2+1$ when $M$ is even and $L=(M+1) / 2$ when $M$ is odd. The $M \times L$ matrix $\mathbf{Q}_{2}(\mathbf{X})$ is the sum of the following four $M \times L$ matrices:

$$
\begin{aligned}
& {\left[\mathbf{W}_{1}\right]_{p q}= \begin{cases}X_{p+q-1}, & p+q \leq M+1, \\
0, & \text { otherwise }\end{cases} } \\
& {\left[\mathbf{W}_{2}\right]_{p q}= \begin{cases}X_{p-q+1}, & p \geq q \geq 2, \\
0, & \text { otherwise }\end{cases} } \\
& {\left[\mathbf{W}_{3}\right]_{p q}= \begin{cases}X_{M+1+p-q}, & p<q \leq l, \\
0, & \text { otherwise }\end{cases} } \\
& {\left[\mathbf{W}_{4}\right]_{p q}= \begin{cases}X_{p+q-M-1}, & 2 \leq q \leq l, p+q \geq M+2, \\
0, & \text { otherwise, }\end{cases} }
\end{aligned}
$$

where $l=M / 2$ for even $M$ and $l=(M+1) / 2$ for odd $M$.

Lemma A.3. For any $M \times 1$ complex vector $\mathbf{X}$ and any $M \times M$ banded complex symmetric Toeplitz matrix $\mathbf{A}$, one has

$$
\mathbf{A} \cdot \mathbf{X}=\mathbf{Q}_{3}(\mathbf{X}) \cdot \mathbf{a},
$$

where the $L \times 1$ vector $\mathbf{a}$ is given by

$$
a_{i}=A_{1 i}, \quad \text { for } i=1,2, \ldots, L
$$

and $L$ is the highest superdiagonal that is different from zero. The $M \times L$ matrix $\mathbf{Q}_{3}(\mathbf{X})$ is given by the sum of the following two $M \times L$ matrices

$$
\begin{aligned}
& {\left[\mathbf{W}_{1}\right]_{p q}= \begin{cases}X_{p+q-1}, & p+q \leq M+1, \\
0, & \text { otherwise }\end{cases} } \\
& {\left[\mathbf{W}_{1}\right]_{p q}= \begin{cases}X_{p-q+1}, & p \geq q \geq 2, \\
0, & \text { otherwise. }\end{cases} }
\end{aligned}
$$




\section{References}

[1] R. O. Schmidt, "Multiple emitter location and signal parameter estimation," IEEE Transactions on Antennas and Propagation, vol. 34, no. 3, pp. 276-280, 1986.

[2] J. Capon, "High-resolution frequency-wavenumber spectrum analysis," Proceedings of the IEEE, vol. 57, no. 8, pp. 1408 $1418,1969$.

[3] B. Friedlander and A. J. Weiss, "Direction finding in the presence of mutual coupling," IEEE Transactions on Antennas and Propagation, vol. 39, pp. 273-284, 1991.

[4] R. B. Ertel, Z. Hu, and J. H. Reed, "Antenna array hardware amplitude and phase compensation using baseband antenna array outputs," in Proceedings of the IEEE 49th Vehicular Technology Conference (VTC '99), pp. 1759-1763, September 1999.

[5] K. R. Dandekar, H. Ling, and G. Xu, "Smart antenna array calibration procedure including amplitude and phase mismatch and mutual coupling effects," in Proceedings of the IEEE International Conference on Personal Wireless Communications, pp. 293-297, December 2000.

[6] B. P. Ng, J. P. Lie, M. H. Er, and A. Feng, "A practical simple geometry and gain/phase calibration technique for antenna array processing," IEEE Transactions on Antennas and Propagation, vol. 57, no. 7, pp. 1963-1972, 2009.

[7] W. Keizer, "Fast and accurate array calibration using a synthetic array approach," IEEE Transactions on Antennas and Propagation, vol. 59, pp. 4115-4122, 2011.

[8] H.-S. Lui, H. T. Hui, and M. S. Leong, "A note on the mutualcoupling problems in transmitting and receiving antenna arrays," IEEE Antennas and Propagation Magazine, vol. 51, no. 5, pp. 171-176, 2009.

[9] K. M. Pasala and E. M. Friel, "Mutual coupling effects and their reduction in wideband direction of arrival estimation," IEEE Transactions on Aerospace and Electronic Systems, vol. 30, no. 4, pp. 1116-1122, 1994.

[10] H. T. Hui, "A practical approach to compensate for the mutual coupling effect in an adaptive dipole array," IEEE Transactions on Antennas and Propagation, vol. 52, no. 5, pp. 1262-1269, 2004.

[11] C. K. E. Lau, R. S. Adve, and T. K. Sarkar, "Minimum norm mutual coupling compensation with applications in direction of arrival estimation," IEEE Transactions on Antennas and Propagation, vol. 52, no. 8, pp. 2034-2041, 2004.

[12] B. Friedlander, "A sensitivity analysis of the MUSIC algorithm," IEEE Transactions on Acoustics, Speech, and Signal Processing, vol. 38, no. 10, pp. 1740-1751, 1990.

[13] W. Kahn and H. Kurss, "Minimum-scattering antennas," IEEE Transactions on Antennas and Propagation, vol. 13, pp. 671675, 1965.

[14] D. H. Johnson and D. E. Dudgeon, Array Signal Processing: Concepts and Techniques, Simon \& Schuster, 1992.

[15] H. L. Van Trees, Optimum Array Processing, Part IV of Detection, Estimation, and Modulation Theory, Wiley-Interscience, New York, NY, USA, 2002.

[16] I. A. S. Milton Abramowitz, Handbook of Mathematical Functions With Formulas, Graphs, and Mathematical Tables, Dover Publications, New York, NY, USA, 1965.

[17] B. H. Wang, H. T. Hui, and M. S. Leong, "Decoupled 2D direction of arrival estimation using compact uniform circular arrays in the presence of elevation-dependent mutual coupling," IEEE Transactions on Antennas and Propagation, vol. 58 , no. 3 , pp. 747-755, 2010.
[18] R. Goossens and H. Rogier, "A hybrid UCA-RARE/RootMUSIC approach for 2-D direction of arrival estimation in uniform circular arrays in the presence of mutual coupling," IEEE Transactions on Antennas and Propagation, vol. 55, no. 3, pp. 841-849, 2007. 

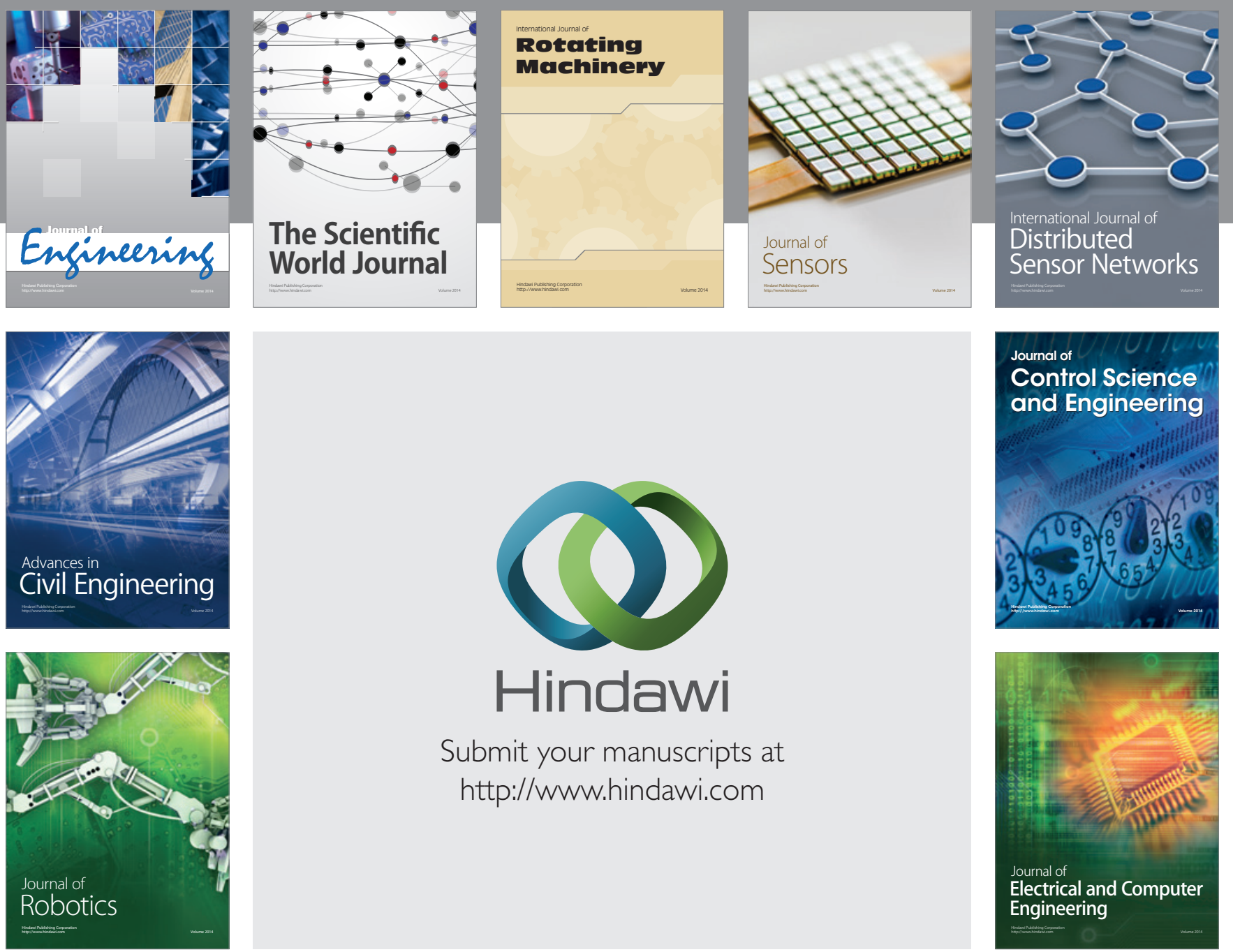

Submit your manuscripts at

http://www.hindawi.com
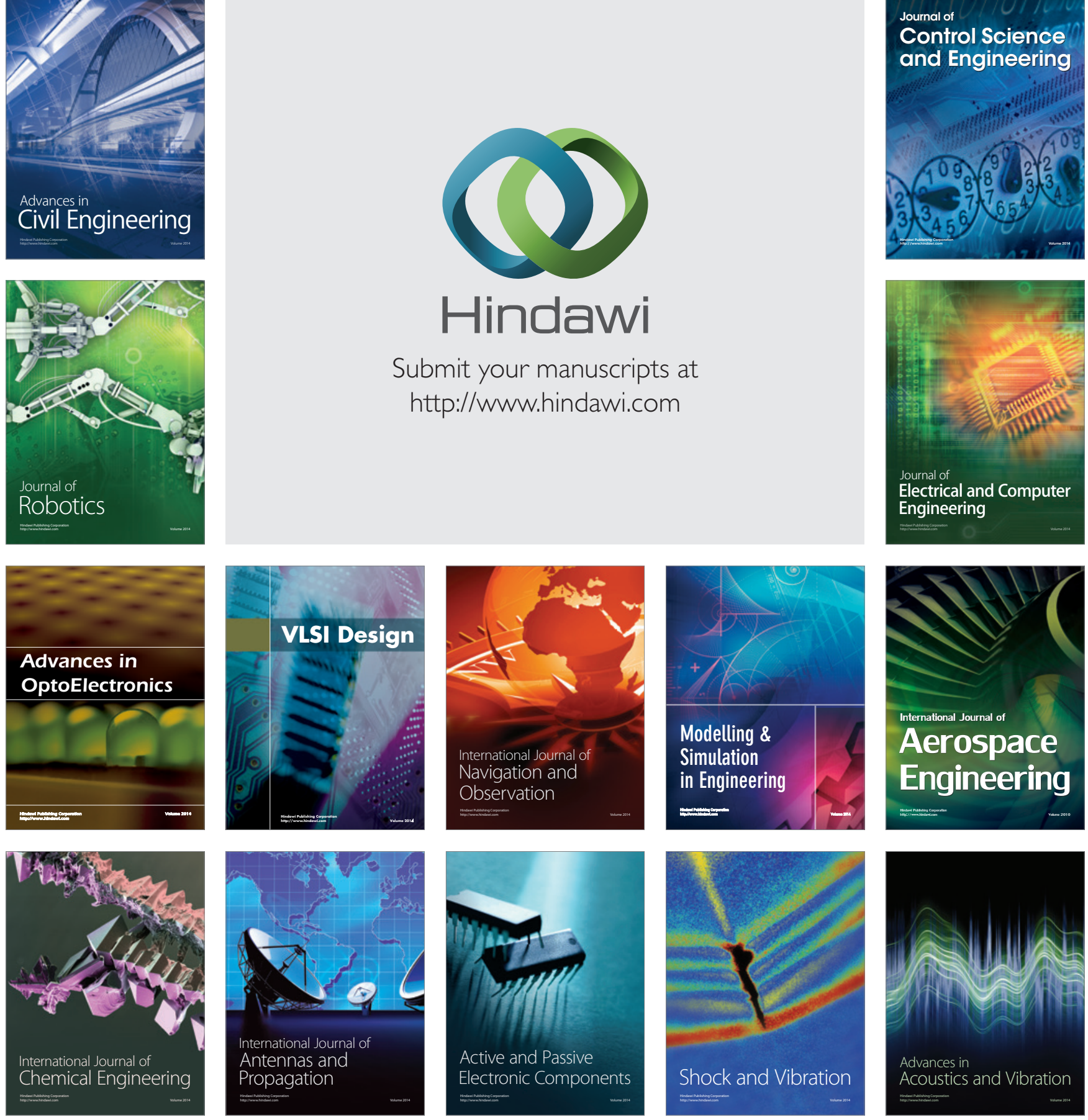